\title{
Stroke Mortality in Kenya's Public Tertiary Hospitals: A Prospective Facility-Based Study
}

\author{
Lydia Kaduka $^{a} \quad$ Erastus Muniu $^{a} \quad$ Chrispine Oduor $^{\mathrm{b}} \quad$ Jane Mbuic \\ Robai Gakunga $^{d}$ Judith Kwasa ${ }^{e}$ Sylvanus Wabwire ${ }^{f}$ Nathan Okerosi ${ }^{c}$ \\ Anne Korirc Scot Remick ${ }^{\mathrm{g}}$ \\ ${ }^{a}$ Centre for Public Health Research, Kenya Medical Research Institute, Nairobi, Kenya; \\ ${ }^{b}$ Department of Medicine, School of Medicine, College of Health Sciences, Moi University, \\ Eldoret, Kenya; ' Centre for Clinical Research, Kenya Medical Research Institute, Nairobi, \\ Kenya; ${ }^{d}$ Kenya Cancer Association, Nairobi, Kenya; ${ }^{e}$ Department of Clinical Medicine and \\ Therapeutics, Kenyatta National Hospital, Nairobi, Kenya; ${ }^{f}$ Kenyatta National Hospital, \\ Nairobi, Kenya; 9 Maine Medical Center Research Institute, Portland, ME, USA
}

\section{Keywords}

Stroke $\cdot$ Mortality $\cdot$ Survival $\cdot$ Kenya $\cdot$ Sub-Saharan Africa

\section{Abstract}

Background: Despite the increasing global burden of stroke, there are limited data on stroke from Kenya to guide in decision-making. Stroke occurrence in sub-Saharan Africa has been associated with poor health outcomes. This study sought to establish the stroke incidence density and mortality in Kenya's leading public tertiary hospitals for purposes of informing clinical practice and policy. Methods: This is a prospective study conducted at Kenya's leading referral hospitals, namely, Kenyatta National Hospital $(\mathrm{KNH})$ and Moi Teaching and Referral Hospital (MTRH). Adult patients with confirmed cases of stroke were recruited from February 2015 to January 2016 and followed up for a minimum period of 1 year. The WHO 2006 Stroke STEPS instrument was used to collect data on incidence and mortality at days 10 and 28 and every 3 months for 24 months. The person-time of follow-up was computed from admission to death, loss to follow-up, or the end of the study. A survival regression analysis was done using the Cox proportional hazards model. Results: A total of 719 patients were recruited (KNH: $n=406$ [56.5\%]; MTRH: $n=313$ [43.5\%]). The mean age was $58.6 \pm 18.7$ years, and the male-to-female ratio was $1: 1.4$. Ischemic stroke accounted for $56.1 \%$ of the stroke cases. The 
peak age for stroke was between 50 and 69 years, when $36.3 \%$ of the cases occurred. Mortality at day 10 and day 28 was 18.4 and $26.7 \%$, respectively. The inpatient mortality rate was $21.6 \%$. The stroke incidence density was 507 deaths per 1,000 person-years of follow-up. The mean survival time was significantly different between inpatients (13.9 months; $95 \% \mathrm{Cl}: 13.0$ 14.7) and outpatients (18.6 months; $95 \% \mathrm{Cl}: 17.2-19.9)(p<0.001)$. A 1-year increase in age increased the hazard by $1.8 \%$. Inpatients had a 3.9 -fold increase in hazard compared to outpatients. Conclusions: Mortality due to stroke is high, with poor survival observed in the first year after stroke. The risk of death increases with increasing age and duration of hospital stay. There is need for attention to quality of care and long-term needs of stroke patients to mitigate the high mortality rates observed. Public health initiatives aimed at early screening and diagnosis should be enhanced. Further research is recommended to establish the true burden of stroke at the community level to inform appropriate mitigation measures.

(c) 2018 The Author(s)

Published by S. Karger AG, Basel

\section{Introduction}

The global burden of stroke is increasing, with $8 \%$ of all first-ever strokes occurring in Africa. There are limited data on stroke from sub-Saharan Africa, with the resultant information deficit hampering evidence-based decision-making. The few studies on stroke in Africa provide a glimpse of poor health outcomes associated with the disease. For instance, inpatient stroke mortality by day 30 has been reported at $19.3 \%$ in the Democratic Republic of Congo [1], 33.3\% in Tanzania, 43.2\% in Ghana, and 23.2\% in Cameroon [2-4]. The 6-month stroke mortality rate in South Africa is $23 \%[5,6]$.

Some of the factors cited as contributing to the increased risk of death include stroke severity, functional disability, high blood pressure, increasing age, dysphagia, and infections such as pneumonia [7-9]. Low survival rates after ischemic stroke have been attributed to lack of access to acute management of strokes, socioeconomic factors, and variations in geographic locations [10]. Female stroke survivors have been found to have a higher probability of survival 1 year after stroke [11].

There is little information on the actual stroke burden in Kenya. Hardly any prospective studies have been done to assess stroke outcomes and survival among patients attended to in Kenyan public hospitals. This study sought to establish the stroke incidence density and mortality in Kenya's leading public tertiary hospitals for purposes of informing policy and practice.

\section{Subjects and Methods}

\section{Study Sites}

The study was conducted at Kenyatta National Hospital (KNH) located in Nairobi, the capital city of Kenya, and Moi Teaching and Referral Hospital (MTRH) in Eldoret, Western Kenya. KNH and MTRH are the largest public tertiary hospitals in Kenya with a bed capacity of 1,800 and 850 , respectively, and they predominantly serve urban and rural populations, respectively.

\section{Study Design and Population}

This is a prospective study among stroke inpatients and outpatients diagnosed and/or attended to at KNH and MTRH. Patients were recruited from February 2015 to January 2016 and followed up for a minimum period of 1 year. 


\section{Criteria for Patient Selection}

The study included adult patients with confirmed cases of stroke based on imaging results. All stroke patients were targeted to minimize the likelihood of selection bias. The combination approach (hot and cold) case finding method was used to identify stroke cases.

\section{Sample Size Estimation}

The sample size was based on the unknown proportion of the most prevalent stroke type (thus, 50\% assumed), a desired precision for the indicator of 5\% and 95\% confidence level. Fisher's formula [12] for estimating the minimum sample size was used, giving a minimum sample of 385. This number was achieved by recruiting stroke patients over a 1-year period, with each hospital contributing a minimum of 200 patients.

\section{Recruitment and Training of Field Staff}

Research assistants with a medical background were recruited and trained on the protocol and study procedures using predesigned data collection tools. The WHO STEPwise Approach to Stroke Surveillance Manual was used for training [13]. Training and certification were done to harmonize data collection methods between researchers. Piloting of the study tools was done, followed by definitive data collection.

\section{Data Collection}

Stroke inpatients were recruited upon admission, while outpatients were recruited from the neurology clinic and at casualty (emergency room department) at both hospitals. Data collected included details on sociodemographics, stroke events, case management, and discharge status using the WHO 2006 Stroke STEPS instrument. The instrument collects in a standardized manner basic epidemiological data on incidence, major risk factors, morbidity and mortality trends, and intervention strategies in recent (acute) stroke. Follow-up entailed assessing clinical outcomes using the modified Rankin Scale, and gathering information on patients' vital status after discharge. The participants recruited during the 1-year period were followed up at intervals of 10 days, 28 days, and every 3 months for 24 months. A complete follow-up was carried out for all the participants for day 10, day 28, 3 months, 6 months, 9 months, and 12 months. For those discharged, follow-up was done through telephone interviews.

\section{Data Management and Analysis}

To ensure the collection of accurate data, a two-level interview form review process was put in place. The data were doubly entered using the Microsoft Access application. Cleaned and validated data were then exported into the IBM Statistical Package for Social Sciences version 21.0 (IBM SPSS) for analysis. The databases (Microsoft Access and IBM SPSS formats) were copied onto external hard disks for backup and storage.

The distribution characteristics for continuous data were confirmed using the Kolmogorov-Smirnov test and exploratory data analysis. The analysis involved descriptive statistics. To test for associations between independent variables and stroke type, for example, the Student $t$ test (normally distributed data) or the Mann-Whitney U test (skewed data) was carried out for continuous variables, and the $\chi^{2}$ test or Fisher's exact probability test, where applicable, to categorical data. The person-time of follow-up of each participant was computed from admission to death, loss to follow-up, or the end of the study, whichever came first. Survival regression analysis was done using the Cox proportional hazards model. 

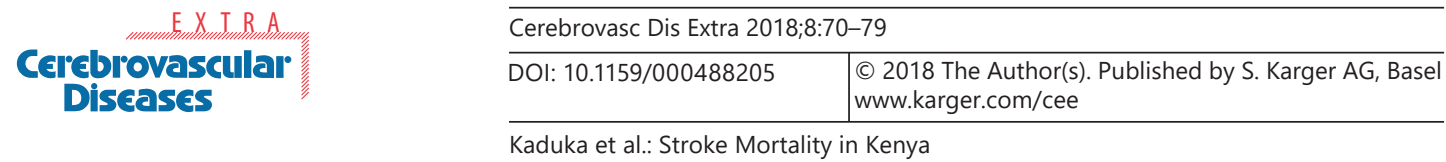

Table 1. Characteristics of the stroke patients in KNH and MTRH

\begin{tabular}{|c|c|c|c|c|}
\hline Characteristics & $\mathrm{KNH}(n=406)$ & MTRH $(n=313)$ & Overall & $p$ value \\
\hline Sex & & & & 0.144 \\
\hline Male & $166(40.9)$ & $145(46.3)$ & $311(43.3)$ & \\
\hline Female & $240(59.1)$ & $168(53.7)$ & $408(56.7)$ & \\
\hline Mean age \pm SD $(95 \% \mathrm{CI})$, years & & & $58.6 \pm 18.7(57.3-60.0)$ & \\
\hline Age group & & & & $<0.001$ \\
\hline$<30$ years & $34(8.4)$ & $11(3.5)$ & $45(6.3)$ & \\
\hline $30-39$ years & $49(12.1)$ & $26(8.3)$ & $75(10.4)$ & \\
\hline $40-49$ years & $63(15.5)$ & $54(17.3)$ & $117(16.3)$ & \\
\hline $50-59$ years & $70(17.2)$ & $50(16)$ & $120(16.7)$ & \\
\hline $60-69$ years & $98(24.1)$ & 43 (13.7) & $141(19.6)$ & \\
\hline $70-79$ years & $48(11.8)$ & $67(21.4)$ & $115(16.0)$ & \\
\hline$\geq 80$ years & $44(10.8)$ & $62(19.8)$ & $106(14.7)$ & \\
\hline Education & & & & $<0.001$ \\
\hline No formal schooling & $62(15.3)$ & $135(43.1)$ & $197(27.4)$ & \\
\hline Primary school & $211(52.0)$ & $111(35.4)$ & $322(44.8)$ & \\
\hline Secondary school completed & 89 (21.9) & $42(13.4)$ & $131(18.2)$ & \\
\hline College/university completed & $36(8.9)$ & $25(8)$ & $61(8.5)$ & \\
\hline Unknown & $8(2.0)$ & $0(0)$ & $8(1.0)$ & \\
\hline Occupation & & & & $<0.001$ \\
\hline Formal employment & $99(24.4)$ & $36(11.6)$ & 135 (18.8) & \\
\hline Self-employed & $177(43.5)$ & $183(58.5)$ & $360(50.0)$ & \\
\hline Student & $8(2.0)$ & $2(0.6)$ & $10(1.4)$ & \\
\hline Homemaker/housewife & $18(4.4)$ & $36(11.5)$ & $54(7.5)$ & \\
\hline Retired & $35(8.6)$ & $11(3.5)$ & $46(6.4)$ & \\
\hline Unemployed & $69(17.0)$ & $45(14.4)$ & $114(15.9)$ & \\
\hline Stroke subtype & & & & 0.002 \\
\hline Hemorrhagic & $199(63.0)$ & $117(37.4)$ & $316(43.9)$ & \\
\hline Ischemic & $207(51.4)$ & $196(48.6)$ & $400(56.1)$ & \\
\hline \multicolumn{5}{|c|}{ Median blood pressure (IQR), mm Hg } \\
\hline Systolic & & & $155(40)$ & \\
\hline Diastolic & & & $90(26)$ & \\
\hline
\end{tabular}

Values are presented as $n(\%)$ unless specified otherwise. KNH, Kenyatta National Hospital; MTRH, Moi Teaching and Referral Hospital.

\section{Results}

A total of 719 patients (KNH: $n=406$ [56.5\%]; MTRH: $n=313$ [43.5\%]) with a mean age of $58.6 \pm 18.7$ years participated in the study. Overall, 311 (43.3\%) were male and $408(56.7 \%)$ female, giving a male-to-female ratio of 1:1.4. The majority (482 [67\%]) were above 50 years of age, with $514(71.5 \%)$ having received formal education and $135(18.8 \%)$ being in formal employment. Ischemic stroke accounted for $56.1 \%$ of the stroke cases. Table 1 shows the sociodemographic and clinical characteristics of the participants by health facility.

The proportion of females with ischemic and with hemorrhagic stroke was 57.6 and $54 \%$, respectively, whereas the respective proportion among men was 42.4 and $46 \%$. The peak age for stroke was between 50 and 69 years, when 261 (36.3\%) of the cases occurred.

\section{Stroke Incidence Density and Mortality}

All participants were followed up for a minimum period of 1 year. Mortality was $18.4 \%$ (ischemic: $15.4 \%$; hemorrhagic: $22.2 \% ; p=0.02$ ) by day 10 and $26.7 \%$ (ischemic: $23.8 \%$; 
Table 2. Distribution of 1-year cumulative mortality by stroke type among patients in KNH and MTRH

\begin{tabular}{lllll}
\hline Follow-up time & \multicolumn{2}{l}{ One-year cumulative mortality by stroke type, $\%$} & \multirow{2}{*}{$p$ value } \\
\cline { 2 - 3 } & $\begin{array}{l}\text { ischemic } \\
(n=403)\end{array}$ & $\begin{array}{l}\text { hemorrhagic } \\
(n=316)\end{array}$ & $\begin{array}{l}\text { total } \\
(n=719)\end{array}$ & \\
\hline 10 days & 15.4 & 22.2 & 18.4 & 0.02 \\
28 days & 23.8 & 30.4 & 26.7 & 0.047 \\
3 months & 33.7 & 34.5 & 34.1 & 0.82 \\
6 months & 39.5 & 35.8 & 37.8 & 0.31 \\
9 months & 43.4 & 36.4 & 40.3 & 0.058 \\
12 months & 44.9 & 37.7 & 41.7 & 0.052 \\
\hline
\end{tabular}

KNH, Kenyatta National Hospital; MTRH, Moi Teaching and Referral Hospital.

Table 3. Distribution of deaths and loss to follow-up by follow-up time among patients at KNH and MTRH

\begin{tabular}{|c|c|c|c|c|c|c|c|}
\hline \multirow{3}{*}{$\begin{array}{l}\text { Follow-up } \\
\text { time }\end{array}$} & \multirow{3}{*}{$\begin{array}{l}\text { Subjects } \\
\text { followed up, } n\end{array}$} & \multicolumn{6}{|c|}{ Status after follow-up time } \\
\hline & & \multicolumn{2}{|c|}{ alive } & \multicolumn{2}{|c|}{ lost to follow-up } & \multicolumn{2}{|l|}{ dead } \\
\hline & & $n$ & $\%$ & $n$ & $\%$ & $n$ & $\%$ \\
\hline 10 days & 719 & 578 & 80.4 & 9 & 1.3 & 132 & 18.4 \\
\hline 28 days & 578 & 515 & 88.6 & 3 & 1.0 & 60 & 10.4 \\
\hline 3 months & 515 & 447 & 86.6 & 15 & 3.1 & 53 & 10.3 \\
\hline 6 months & 447 & 414 & 92.6 & 6 & 1.3 & 27 & 6.0 \\
\hline 9 months & 417 & 394 & 93.8 & 5 & 1.9 & 18 & 4.3 \\
\hline 12 months & 394 & 363 & 92.1 & 21 & 5.3 & 10 & 2.5 \\
\hline 15 months & 300 & 295 & 98.3 & 1 & 0.3 & 4 & 1.3 \\
\hline 18 months & 220 & 211 & 95.9 & 4 & 1.8 & 5 & 2.3 \\
\hline 21 months & 104 & 98 & 94.2 & 6 & 5.8 & 0 & - \\
\hline 24 months & 3 & 3 & 100 & 0 & - & 0 & - \\
\hline \multicolumn{4}{|c|}{ Total (out of 719) } & 67 & 9.3 & 309 & 42.97 \\
\hline
\end{tabular}

KNH, Kenyatta National Hospital; MTRH, Moi Teaching and Referral Hospital.

hemorrhagic: $30.4 \% ; p=0.05$ ) by day 28 , as shown in Table 2 . The cumulative mortality rate by month 3 was $245(34.1 \%)$, by month 6 it was 272 (37.8\%), by month 9 it was $290(40.3 \%)$, and by month 12 it was 300 (41.7\%). High mortality was recorded in the first 3 months of follow-up ( $18.4 \%$ by day $10,10.4 \%$ by day 28 , and $10.3 \%$ by month 3 , respectively); mortality then decreased with the increase in follow-up time, as shown in Table 3. The stroke incidence density was 507 deaths per 1,000 person-years of follow-up.

\section{Stroke Survival by Sex, Stroke Type, Facility, and Inpatient/Outpatient Status}

The mean survival time was 15.3 months (95\% CI: 14.1-16.5) among males and 14.2 months (95\% CI: 13.2-15.3) among females, which was not statistically significantly different ( $p=0.16$ ). The mean survival time was 14.2 months (95\% CI: 13.1-15.2) among patients with ischemic stroke and 15.4 months (95\% CI: 14.1-16.6) among patients with hemorrhagic stroke ( $p=0.12$ ). By site, the mean survival time was 15.2 months (95\% CI: 14.1-16.3) among stroke patients at KNH and 12.5 months (95\% CI: 11.4-13.5) among stroke patients at MTRH ( $p=0.14)$. During the 24 -month study period, 639 patients $(88.9 \%)$ were admitted, of whom 
Fig. 1. Mortality rate by stroke type among patients at Kenyatta National Hospital and Moi Teaching and Referral Hospital ( $p=$ 0.12 ).

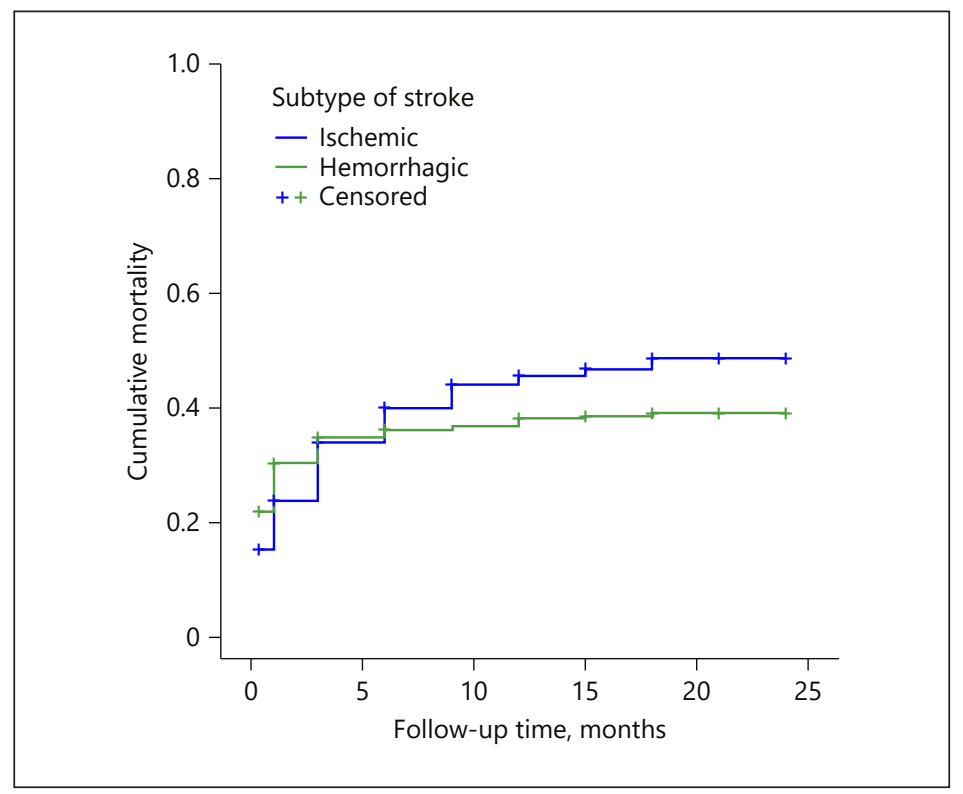

Fig. 2. Mortality rate by sex among patients at Kenyatta National Hospital and Moi Teaching and Referral Hospital ( $p=0.16$ ).

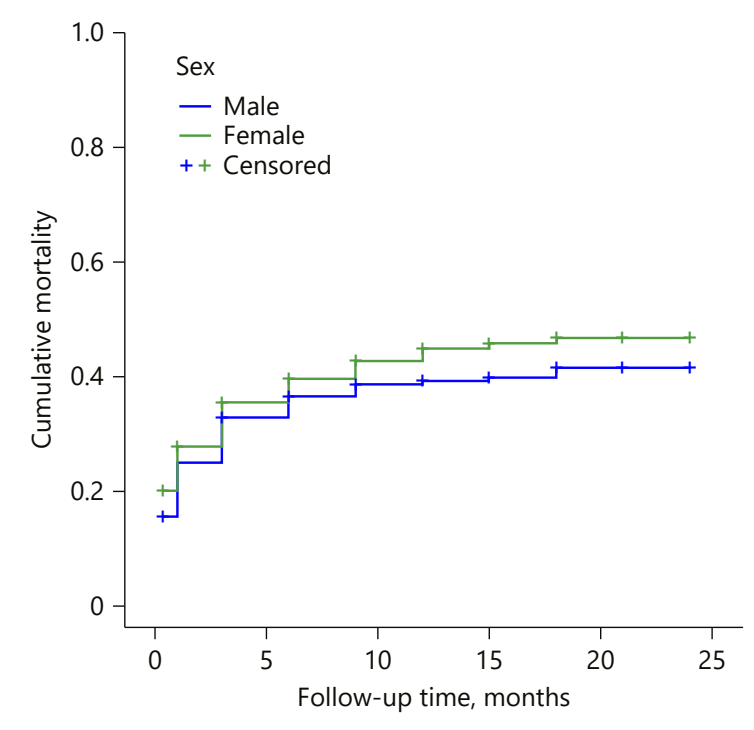

$387(60.6 \%)$ spent less than 10 days in hospital. The inpatient mortality rate was $21.6 \%$. Figures 1-4 show the mortality rates by stroke type, sex, health facility, and inpatient/outpatient status, respectively. A significant difference $(p<0.001)$ in survival time was observed between inpatients (13.9 months; $95 \% \mathrm{CI}$ : $13.0-14.7)$ and outpatients (18.6 months; $95 \% \mathrm{CI}$ : 17.2-19.9).

The survival regression analysis showed that a 1-year increase in age increased the hazard by $1.8 \%$, and that inpatients had a 3.9 -fold increase in hazard compared to outpatients. Figure 5 shows the stroke mortality function after adjustment for sex, age, stroke type, inpatient/outpatient status, and health facility. 
Fig. 3. Mortality by health facility among patients at Kenyatta National Hospital (KNH) and Moi Teaching and Referral Hospital (MTRH) $(p=0.14)$.

Fig. 4. Mortality rate by inpatient/outpatient status among patients at Kenyatta National Hospital and Moi Teaching and Referral Hospital $(p<0.001)$.
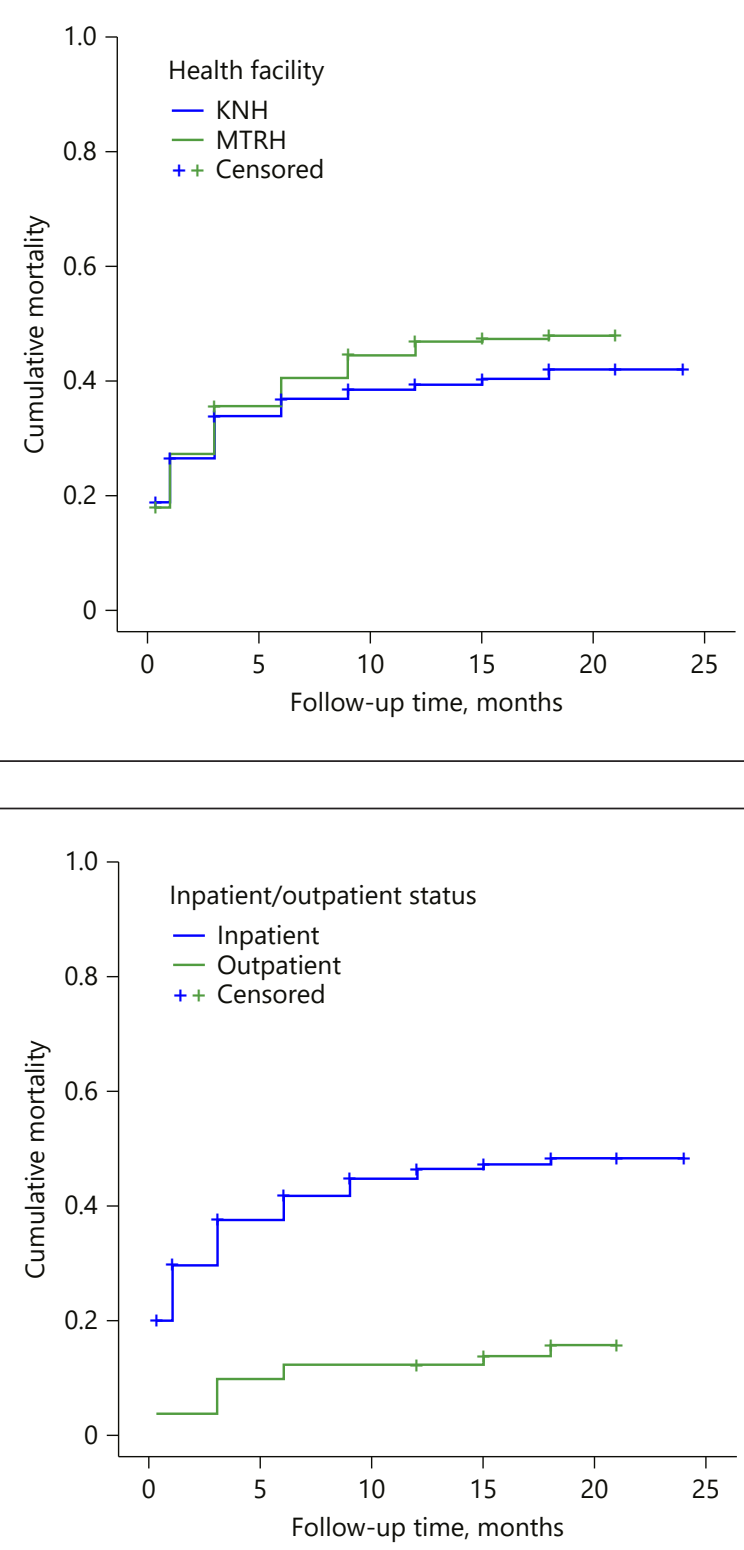

\section{Discussion}

Our study shows a high proportion of ischemic strokes overall, with women being affected more strongly and men surviving longer. The stroke incidence density and mortality remain high in the current setup. Hemorrhagic stroke contributed the most to mortality by day 10 and day 28. There were significant differences in patient survival, with outpatients surviving longer than inpatients. The risk of death increased with increasing age and inpatient status.

This is the first prospective study that provides estimates of stroke incidence density and survival in Kenya's largest public tertiary hospitals. The high inpatient mortality found has previously been observed in Kenya, Uganda, Ghana, and Nigeria [14-17]. The quality of care and performance of public health facilities in the management of stroke are factors that 


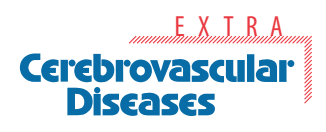

Fig. 5. Stroke mortality function at the mean of the covariates (after adjustment for sex, age, stroke type, inpatient/outpatient status, and health facility).

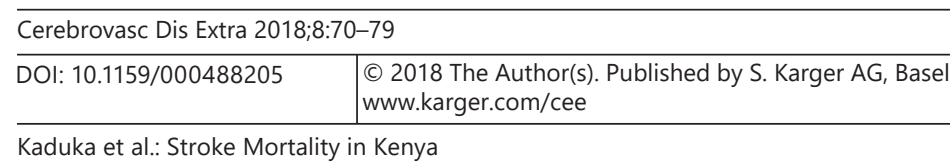

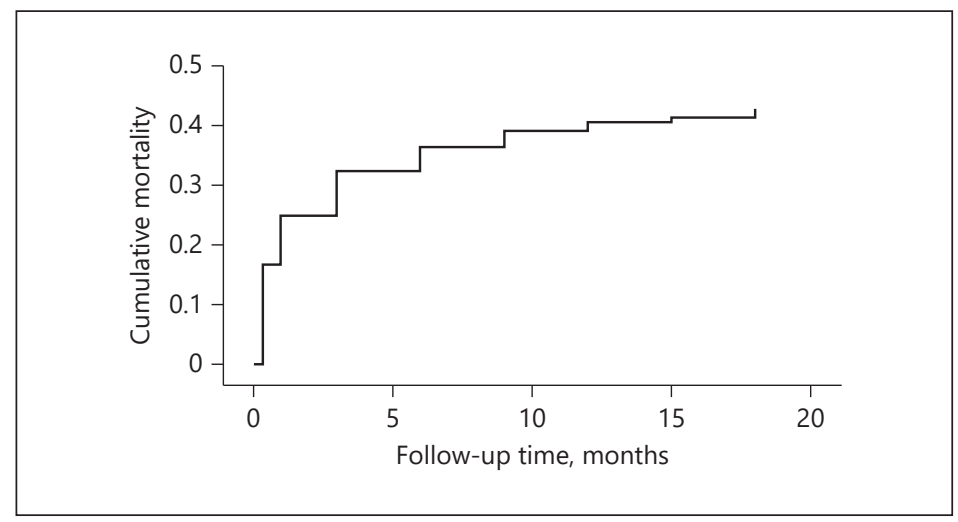

require further examination. There is also a need to assess the geographical distribution of and access to acute stroke care services in Kenya to guarantee a reliable and efficient system of stroke care $[10,18]$. Time to treatment is an important prognostic factor for outcome, and investment in specialized stroke treatment centers is key [19]. Our findings also support the reported impact of stroke severity on short-term but not so much on long-term survival [20], with differences in survival observed between the stroke types. Close to two-thirds of the patients spent less than 10 days in hospital. The role of the community in providing long-term care should therefore be explored and appropriate capacities developed.

The finding on differences in stroke mortality by sex contradicts results reported from developed nations, and further supports the need for further research on stroke epidemiology in developing countries [21]. Stroke mortality was higher among women than among men. As has been previously demonstrated for other sub-Saharan African countries, there were notable differences in survival, with men surviving longer than women [11,22].

There is a need, therefore, for (1) strengthening prehospital stroke care to ensure rapid transfer to the hospital and early management; (2) improvement of in-hospital stroke care taking into consideration the established stroke quality-of-care indicators; and (3) investment in poststroke rehabilitation, since the majority of patients may not have access to acute stroke therapy (thrombolysis/device embolectomy) and may end up with severe disability.

\section{Study Limitations}

This is a (referral) hospital-based study, and we may not have recruited a representative sample of stroke patients in Kenya, as less severe cases of stroke may not have been admitted or referred for further management (referral bias). Thus, milder forms of ischemic stroke and stroke deaths within the community may have been missed. Our study essentially focused on types of stroke and survival outcome; we did not characterize poststroke functional status. These later observations will be helpful to identify ways forward for rehabilitation. Monitoring of stroke incidence, management, and outcomes across the continuum of care would be the preferred option.

\section{Conclusions}

The current findings provide evidence on the stroke burden and outcomes from a lowand middle-income country. Mortality due to stroke is high, with poor survival in the first year after stroke. Men have favorable outcomes compared to women. The risk of death is increased 
with increasing age and inpatient status. As a way forward, there is a need to develop customized stroke care protocols and to address quality of care and the long-term needs of stroke patients in Kenya. Public health initiatives aimed at early screening and diagnosis should be advanced. Further research and surveillance are encouraged to establish the true burden of stroke at the community level and to institute mitigation measures.

\section{Acknowledgements}

This research was supported by an NIH grant (D43 TW009333) from the Fogarty International Center for the "Cancer and Tobacco Control Training and Research across the Lifespan in Kenya" project spearheaded by Prof. Scot C. Remick of Tufts University, Medford, MA, USA. We wish to thank the Director of KEMRI and the KNH and MTRH Review Committees for granting permission and providing an enabling environment to undertake this study. Many thanks to our research assistants Isaac Kisiangani, Mercy Chepkirui, Yvonne Opanga, Doreen Njeri, Ayub Alembi, Henry Mwangi, and Meinard Shikhang'a, as well as the KNH and MTRH medical fraternity, for their continued support.

\section{Statement of Ethics}

The study protocol was approved by the KEMRI Scientific and Ethics Review Committee (SSC No. 2851), the MTRH Institutional Research and Ethics Committee (IREC/2014/213 Approval No. 0001279), and the KNH/University of Nairobi Ethical Review Committee (Study Registration No. MED/029/2015). The study participants gave their informed consent prior to study recruitment.

\section{Disclosure Statement}

The authors have no conflicts of interest to declare.

\section{Author Contributions}

L. Kaduka and E. Muniu contributed to study conceptualization, implementation, data analysis, and manuscript writing. C. Oduor, J. Mbui, R. Gakunga, J. Kwasa, N. Okerosi, A. Korir, and S. Remick contributed to study conceptualization, implementation, and manuscript writing. S. Wabwire contributed to study implementation and manuscript writing.

\section{References}

1 Limbole EB, Magne J, Lacroix P: Stroke characterization in Sun [sic!] Saharan Africa: Congolese population. Int J Cardiol 2017;240:392-397.

-2 Okeng'o K, Chillo P, Gray WK, Walker RW, Matuja W: Early mortality and associated factors among patients with stroke admitted to a large teaching hospital in Tanzania. J Stroke Cerebrovasc Dis 2017;26:871-878.

-3 Agyemang C, Attah-Adjepong G, Owusu-Dabo E, De-Graft Aikins A, Addo J, Edusei AK, Nkum BC, Ogedegbe G: Stroke in Ashanti region of Ghana. Ghana Med J 2012;46(suppl):12-17.

-4 Nkoke C, Lekoubou A, Balti E, Kengne AP: Stroke mortality and its determinants in a resource-limited setting: a prospective cohort study in Yaounde, Cameroon. J Neurol Sci 2015;358:113-117. 
5 de Villiers L, Badri M, Ferreira M, Bryer A: Stroke outcomes in a socio-economically disadvantaged urban community. S Afr Med J 2011;101:345-348.

6 Wasserman S, de Villiers L, Bryer A: Community-based care of stroke patients in a rural African setting. S Afr Med J 2009;99:579-583.

7 Walker RW, Wakefield K, Gray WK, Jusabani A, Swai M, Mugusi F: Case-fatality and disability in the Tanzanian Stroke Incidence Project cohort. Acta Neurol Scand 2016;133:49-54.

-8 Zenebe G, Alemayehu M, Asmera J: Characteristics and outcomes of stroke at Tikur Anbessa Teaching Hospital, Ethiopia. Ethiop Med J 2005;43:251-259.

-9 Walker RW, McLarty DG, Kitange HM, Whiting D, Masuki G, Mtasiwa DM, Machibya H, Unwin N, Alberti KG: Stroke mortality in urban and rural Tanzania. Adult Morbidity and Mortality Project. Lancet 2000;355:16841687.

-10 Hoffmeister L, Lavados PM, Murta-Nascimento C, Araujo M, Olavarría VV, Castells X: Short- and long-term survival after stroke in hospitalized patients in Chile: a nationwide 5-year study. J Stroke Cerebrovasc Dis 2013;22:e463-e469.

-11 Andersen MN, Andersen KK, Kammersgaard LP, Olsen TS: Sex differences in stroke survival: 10-year followup of the Copenhagen Stroke Study cohort. J Stroke Cerebrovasc Dis 2005;14:215-220.

12 Fisher RA: Statistical Methods for Research Workers. Edinburgh, Oliver and Boyd, 1954

13 World Health Organization: WHO STEPS Stroke Manual: the WHO STEPwise Approach to Stroke Surveillance. Geneva, World Health Organization, 2006.

14 Oduor CO, Keter A, Dieor LO, Siika AM, Williams LS: Stroke types, risk factors, quality of care and outcomes at a referral hospital in Western Kenya. East Afr Med J 2015;92:7.

15 Nakibuuka J, Sajatovic M, Nankabirwa J, Ssendikadiwa C, Furlan AJ, Katabira E, Kayima J, Kalema N, ByakikaTusiime J, Ddumba E: Early mortality and functional outcome after acute stroke in Uganda: prospective study with 30 day follow-up. Springerplus 2015;4:450.

16 Sarfo FS, Acheampong JW, Appiah LT, Oparebea E, Akpalu A, Bedu-Addo G: The profile of risk factors and in-patient outcomes of stroke in Kumasi, Ghana. Ghana Med J 2014;48:127-134.

17 Ekeh B, Ogunniyi A, Isamade E, Ekrikpo U: Stroke mortality and its predictors in a Nigerian teaching hospital. Afr Health Sci 2015;15:74-81.

18 Zweifler RM: Initial assessment and triage of the stroke patient. Prog Cardiovasc Dis 2017;59:527-533.

19 Fuller A, Tran T, Muhumuza M, Haglund MM: Building neurosurgical capacity in low and middle income countries. eNeurologicalSci 2016;3:1-6.

20 Andersen KK, Olsen TS: One-month to 10-year survival in the Copenhagen Stroke Study: interactions between stroke severity and other prognostic indicators. J Stroke Cerebrovasc Dis 2011;20:117-123.

21 Barker-Collo S, Bennett DA, Krishnamurthi RV, Parmar P, Feigin VL, Naghavi M, Forouzanfar MH, Johnson CO, Nguyen G, Mensah GA, Vos T, Murray CJ, Roth GA; GBD 2013 Writing Group; GBD 2013 Stroke Panel Experts Group: Sex differences in stroke incidence, prevalence, mortality and disability-adjusted life years: results from the Global Burden of Disease Study 2013. Neuroepidemiology 2015;45:203-214.

-22 Heikinheimo T, Chimbayo D, Kumwenda JJ, Kampondeni S, Allain TJ: Stroke outcomes in Malawi, a country with high prevalence of HIV: a prospective follow-up study. PLoS One 2012;7:e33765. 